Please do not remove this page

RMIT

UNIVERSITY

\title{
Soft exfoliation of 2D SnO with size-dependent optical properties
}

Singh, Mandeep; Della Gaspera, Enrico; Ahmed, Taimur; Walia, Sumeet; Ramanathan, Rajesh; van Embden, Joel; Mayes, Edwin

https://researchrepository.rmit.edu.au/esploro/outputs/9921860907801341/filesAndLinks?institution=61RMIT_INST\&index=null

Singh, M., Della Gaspera, E., Ahmed, T., Walia, S., Ramanathan, R., van Embden, J., Mayes, E., \& Bansal, V. (2017). Soft exfoliation of 2D SnO with size-dependent optical properties. 2D Materials, 4(2), 1-10. https://doi.org/10.1088/2053-1583/aa6efc

Document Version: Accepted Manuscript

Published Version: https://doi.org/10.1088/2053-1583/aa6efc

Repository homepage: https://researchrepository.rmit.edu.au

CC BY-NC-ND V4.0

(C) 2017 IOP Publishing Ltd

Downloaded On 2023/04/26 21:09:15 +1000

Please do not remove this page 


\section{Soft Exfoliation of 2D SnO with size-dependent}

\section{optical properties}

Mandeep Singh ${ }^{1, \dagger}$, Enrico Della Gaspera ${ }^{1, \dot{t}^{*}}$, Taimur Ahmed ${ }^{2}$, Sumeet Walia ${ }^{2}$, Rajesh Ramanathan ${ }^{1}$, Joel van Embden ${ }^{1}, E_{\text {Ewin Mayes }}^{3}$, Vipul Bansal ${ }^{1, *}$

${ }^{1}$ Ian Potter NanoBioSensing Facility, NanoBiotechnology Research Laboratory, School of Science, RMIT University, Melbourne VIC 3000, Australia

${ }^{2}$ Functional Materials and Microsystems Research Group and Micro Nano Research Facility, RMIT University, Melbourne VIC 3000, Australia

${ }^{3}$ RMIT Microscopy and Microanalysis Facility, School of Science, RMIT University, Melbourne VIC 3000, Australia

\section{Corresponding Authors}

*Email: enrico.dellagaspera@rmit.edu.au (EDG); vipul.bansal@rmit.edu.au (VB)

\section{Keywords}

Two-dimensional material, tin monoxide, liquid phase exfoliation, metal oxides, p-type, quantum confinement. 


\begin{abstract}
Two-dimensional (2D) materials have recently gained unprecedented attention as potential candidates for next-generation (opto)electronic devices due to their fascinating optical and electrical properties. Tin monoxide, $\mathrm{SnO}$, is an important $p$-type semiconductor with applications across photocatalysis (water splitting) and electronics (transistors). However, despite its potential in several important technological applications, $\mathrm{SnO}$ remains underexplored in its 2D form. Here we present a soft exfoliation strategy to produce $2 \mathrm{D} \mathrm{SnO}$ nanosheets with tunable optical and electrical properties. Our approach involves the initial synthesis of layered $\mathrm{SnO}$ microspheres, which are readily exfoliated through a low-power sonication step to form high quality $\mathrm{SnO}$ nanosheets. We demonstrate that the properties of 2D $\mathrm{SnO}$ are strongly dependent on its dimensions. As verified through optical absorption and photoluminescence studies, a strong size-dependent quantum confinement effect in 2D $\mathrm{SnO}$ leads to substantial variation in its optical and electrical properties. This results in a remarkable $(>1 \mathrm{eV})$ band gap widening in atomically thin $\mathrm{SnO}$. Through photoconductivity measurements, we further validate a strong correlation between the quantum-confined properties of $2 \mathrm{D} \mathrm{SnO}$ and the selective photoresponse of atomically thin sheets in the high energy UV light. Such tunable semiconducting properties of $2 \mathrm{D} \mathrm{SnO}$ could be exploited for a variety of applications including photocatalysis, photovoltaics and optoelectronics in general.
\end{abstract}




\section{Introduction}

Two-dimensional (2D) materials have recently emerged as a novel class of compounds with unique electrical, optical and mechanical properties. ${ }^{1}$ They constitute a paradigm shift in the way we conceive and design nanomaterial-based devices, and they are predicted to revolutionize current electronic device technologies. ${ }^{2}$ Following the pioneering work on both mechanically exfoliated ${ }^{3}$ and liquid-phase exfoliated graphene, ${ }^{4}$ many $2 \mathrm{D}$ materials have been synthesized, including transition metal dichalcogenides (TMDC, such as $\mathrm{MoS}_{2}, \mathrm{MoSe}_{2}, \mathrm{WS}_{2}$, $\mathrm{WSe}_{2}, \mathrm{NbS}_{2}$ ), ${ }^{5,6}$ metal oxides and hydroxides (such as $\mathrm{MoO}_{3}, \mathrm{MnO}, \mathrm{WO}_{3}, \mathrm{Ni}(\mathrm{OH})_{2}$ and many ternary and quaternary compounds), ${ }^{7,8}$ metal nitrides and carbides (such as hexagonal BN and the family of compounds known as MXenes), ${ }^{9}$ elemental compounds (including phosphorene, antimonene and silicene), ${ }^{10,11}$ and cesium lead halide perovskites. ${ }^{12}$

Although these classes of compounds all have their distinctive properties and applications, metal oxides are particularly interesting because they are environmentally stable, relatively easy to fabricate, and typically comprised of non-toxic, naturally occurring elements, which make them even more interesting for potential large scale production. Two-dimensional metal oxides have also been proven successful as active materials in many applications including energy storage, ${ }^{7}$ transistors, ${ }^{13}$ photodetectors, ${ }^{14}$ photovoltaics, ${ }^{15}$ water splitting, ${ }^{16}$ plasmonics ${ }^{17}$ and sensing. ${ }^{18}$

$\mathrm{SnO}$ is an important $p$-type semiconductor, with very interesting optoelectronic properties, including a small indirect band gap of $\sim 0.7 \mathrm{eV}$, a much larger direct band gap of

$\sim 2.7 \mathrm{eV}$, and high hole mobility. ${ }^{19,20} \mathrm{SnO}$ is also a rare example of an oxide semiconductor in which ambipolar conductivity can be obtained, as it simultaneously offer reasonably large electron affinity (3.7 eV - suitable for n-doping) and reasonably small ionization potential (4.4 $\mathrm{eV}$ - suitable for p-doping). Due to these properties, $\mathrm{SnO}$ has been used to fabricate high 
mobility $p$-type 19,21 $^{10}$ ambipolar ${ }^{22}$ thin film transistors, efficient photocatalysts for water splitting, ${ }^{23}$ anodes for lithium ion batteries, ${ }^{24}$ and, just recently, improved anodes for sodium ion batteries. ${ }^{25}$ Moreover, bulk $\mathrm{SnO}$ has a tetragonal crystalline structure which is layered along the $(00 z)$ crystallographic direction. The layered nature of the crystalline lattice makes $\mathrm{SnO}$ highly promising as a $2 \mathrm{D}$ material. ${ }^{26}$ However, despite its scientific and technological importance, $\mathrm{SnO}$ has hardly been reported in a 2D form. Only a few studies have shown the fabrication of 2D nanosheets of $\mathrm{SnO}$ using either time-consuming hydrothermal and intercalation treatments, ${ }^{21,25}$ or using expensive and lengthy vacuum-based methods such as pulsed laser deposition. ${ }^{23}$ Liquid phase exfoliation methods are promising thanks to their inherent simplicity, and as such have been employed for the synthesis of many 2D materials, although they usually require lengthy and energy-intensive ultrasonication steps. ${ }^{27,28}$ In this study we present the "soft" liquid-phase exfoliation of layered $\mathrm{SnO}$ microspheres to produce 2D SnO nanosheets with tunable optical and electronic properties. By developing a strategy to synthesize highly layered $\mathrm{SnO}$ microspheres, we were able to obtain $\mathrm{SnO}$ nanosheets in just a few minutes through the simple exfoliation of these microspheres in ethanol using a standard low-power $(40 \mathrm{~W})$ bench-top ultrasonic bath. Our method produces highly crystalline, atomically thin and free-standing $\mathrm{SnO}$ nanosheets with lateral dimensions typically between 0.5 and $5 \mu \mathrm{m}$. We demonstrate that large, thick sheets of 2D SnO show bulk-like optical and electronic properties, whereas small, thin 2D SnO displays optical properties consistent with a highly quantum-confined material. We envisage that the ability to tune the properties of 2D $\mathrm{SnO}$ nanosheets according to their thickness will have important technological implications for the fabrication of highly efficient optoelectronic devices.

\section{Results and Discussion}


Our approach to synthesize 2D $\mathrm{SnO}$ nanosheets uses a "soft" liquid phase exfoliation strategy, which is both reproducible and facile. Firstly, we developed and optimized a robust method for preparing layered $\mathrm{SnO}$ microspheres through the thermal decomposition of a tin oxide hydroxide intermediate at moderate temperatures (see Materials and Methods). Briefly, a tin precursor (tin(II) 2-ethylhexanoate) is first reacted with triethylamine at room temperature (see FTIR in Supporting Information, Figure S1). This mixture is then injected into water, causing the instantaneous precipitation of tin oxide hydroxide $\left(\mathrm{Sn}_{6} \mathrm{O}_{4}(\mathrm{OH})_{4}\right)$ as a white powder. Subsequent heating of this powder in an ambient environment at $180{ }^{\circ} \mathrm{C}$ causes its thermal decomposition into $\mathrm{SnO}$ in the form of a black powder (Figure 1), as confirmed by thermogravimetric analysis (TGA) and x-ray diffraction (XRD). TGA shows $\sim 4.2 \%$ weight loss which corresponds to the full dehydration and decomposition of the initial oxide hydroxide to $\mathrm{SnO}$ (Supporting Information, Figure S2). XRD also shows full conversion of $\mathrm{Sn}_{6} \mathrm{O}_{4}(\mathrm{OH})_{4}$ (ICDD No. 84-2157) to SnO (ICDD No. 85-0423) after annealing at $180{ }^{\circ} \mathrm{C}$ (Figure 1). All diffraction peaks detected in the annealed sample can be ascribed to tetragonal $\mathrm{SnO}$, and no residual impurities coming from the initial oxide hydroxide phase could be detected. Scanning electron microscopy (SEM) images of the as-synthesized tin oxide hydroxide and of the annealed $\mathrm{SnO}$ show a clear change in morphology from featureless powders to porous layered microspheres with diameters in the range of 5-20 $\mu \mathrm{m}$ (Figure 1b-e and Supporting Information, Figure S3). We note that a similar morphology of layered $\mathrm{SnO}$ microspheres was recently obtained through a biomimetic approach. ${ }^{29} \mathrm{SEM}$ analysis of our $\mathrm{SnO}$ microspheres indeed confirms their lamellar structure, which is conducive to the formation of thin $\mathrm{SnO}$ nanosheets upon subsequent exfoliation. 

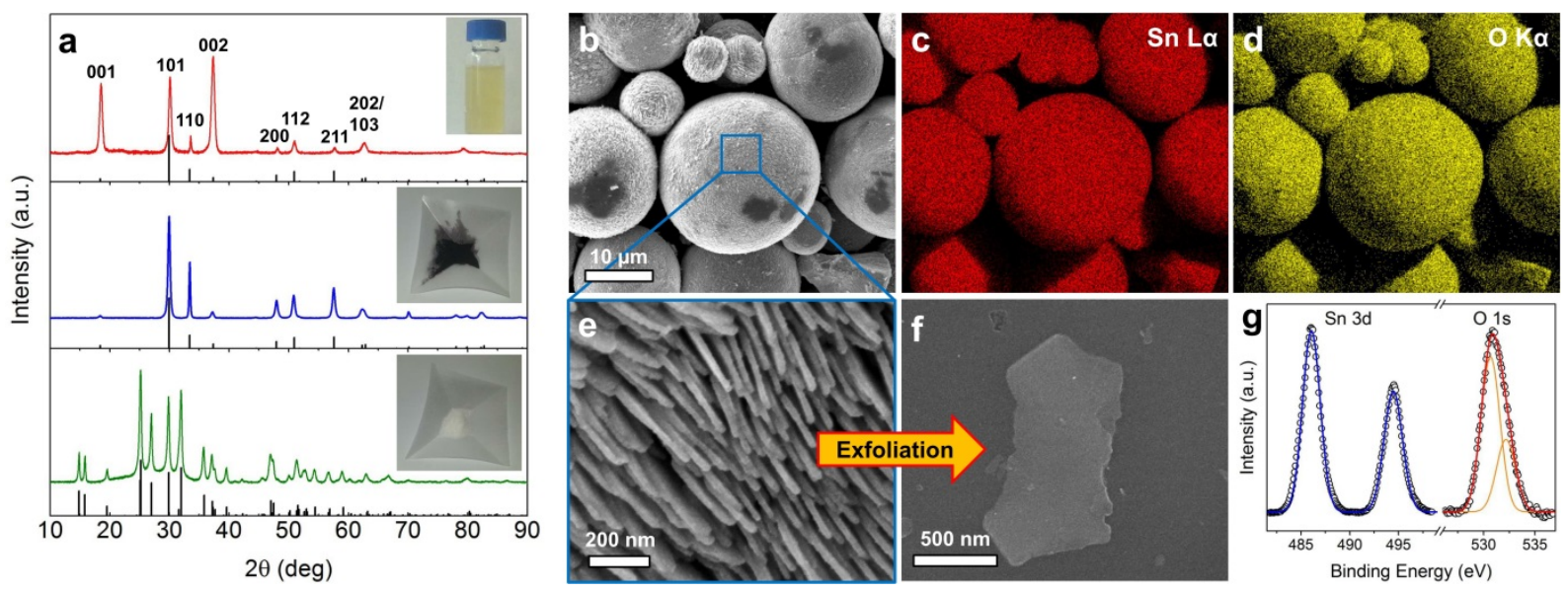

Figure 1. a) XRD patterns of $\mathrm{Sn}_{6} \mathrm{O}_{4}(\mathrm{OH})_{4}$ powders (bottom, green line), layered $\mathrm{SnO}$ microspheres (center, blue line) and exfoliated 2D SnO (top, red line). The main diffraction peaks of $\mathrm{Sn}_{6} \mathrm{O}_{4}(\mathrm{OH})_{4}$ and $\mathrm{SnO}$ are assigned according to the reference ICDD card No. 84-2157 and No. 85-0423, respectively. The insets show pictures of the prepared materials. b-e) SEM analyses and corresponding EDX maps of the SnO powders showing the distinctive layered microsphere morphology. f) SEM of a single $\mathrm{SnO}$ nanosheet obtained after exfoliation. g) XPS characterization of the $\mathrm{Sn} 3 \mathrm{~d}$ and $\mathrm{O} 1$ s core levels for exfoliated $\mathrm{SnO}$ nanosheets.

The as-prepared $\mathrm{SnO}$ microspheres are then dispersed in common solvents (including ethanol and N,N-Dimethylformamide, DMF), and through a simple sonication treatment for a short period of time (1-5 minutes) in a standard bench-top ultrasonic bath a yellow colloidal suspension of exfoliated nanosheets is produced (Figure 1a). This facile exfoliation is in stark contrast with other commonly employed liquid-phase exfoliation techniques currently used to synthesize 2D materials, which involve lengthy (several hours) and powerful (hundreds of Watts) ultrasonication treatments. ${ }^{28}$ The ability of our method to easily exfoliate $\mathrm{SnO}$ nanosheets is ascribed to the distinctive lamellar morphology of the synthesized $\mathrm{SnO}$ microspheres. XRD analysis of the exfoliated sample deposited on glass substrate shows pure $\mathrm{SnO}$ phase, with a preferential orientation along the $(00 z)$ direction, as evidenced by the increase in relative intensity of the (001) and (002) diffraction peaks, and the strong reduction in the intensity of the (110) and (220) peaks (Figure 1a and Supporting Information, Figure 
S4). This suggests that the exfoliated nanosheets lay flat on the substrate exposing their $(00 z)$ surfaces parallel to the substrate. Calculation of the texture coefficient and of the alignment factor (see Supporting Information, Table S1) shows that the extent of orientation is greater than $55 \%$ in the exfoliated sample. ${ }^{30}$ Conversely, the as-synthesized $\mathrm{SnO}$ microspheres, although layered, are randomly oriented during XRD measurements, as expected.

To further analyze the morphology of the exfoliated SnO, we carried out SEM analyses of drop-casted samples of the nanosheet suspensions (Figure 1f and Supporting Information, Figure S5). SnO nanosheets can be clearly seen, with lateral dimension predominantly in the $0.5-1 \mu \mathrm{m}$ range, consistent with the average lateral size of the platelets composing the initial $\mathrm{SnO}$ microspheres (Figure 1e). However, both larger $(2-5 \mu \mathrm{m})$ and smaller $(<500 \mathrm{~nm})$ nanosheets are also observed (as also evident later from TEM images). It should be noted that the smaller nanosheets may result from the fracture and/or further exfoliation of the $\mathrm{SnO}$ platelets during sonication. The different electron contrast of the nanosheets in the electron microscopy images is indicative of their different thicknesses.

As discussed earlier, XRD analysis of the exfoliated material showed the absence of additional species other than $\mathrm{SnO}$ (as for example, metallic $\mathrm{Sn}$ or $\mathrm{SnO}_{2}$ ). To ensure that no surface oxidation/reduction of $\mathrm{SnO}$ occurred during the exfoliation process, the chemical nature of the surface of exfoliated $\mathrm{SnO}$ was probed using $\mathrm{X}$-ray photoelectron spectroscopy (XPS, Figure 1g). The core level spectrum of $\mathrm{Sn} 3 \mathrm{~d}$ shows the characteristic $\mathrm{Sn} 3 \mathrm{~d}_{5 / 2}$ and $\mathrm{Sn}$ $3 \mathrm{~d}_{3 / 2}$ splitting components (spin-orbit splitting of $8.5 \mathrm{eV}$ ) with sharp peaks observed at binding energies of 486.1 and $494.6 \mathrm{eV}$, which correspond to $\mathrm{Sn}^{2+}$ in pure tetragonal $\mathrm{SnO}^{31}$ Additionally, the $\mathrm{O}$ 1s spectrum shows two components with the lower energy feature corresponding to oxygen atoms within the crystalline lattice, while the higher energy 
component corresponds to surface oxygen atoms, including hydroxyl groups and adsorbed organics. ${ }^{32,33}$ All these values are in good agreement with literature data for crystalline SnO. ${ }^{31,34}$

Transmission electron microscopy (TEM) was also used to characterize the morphology and crystallinity of the exfoliated SnO. In agreement with SEM, nanosheets with lateral dimension ranging between hundreds of nanometers and few micrometers are readily observed (Figure 2). In addition, both thick (with high electron contrast) and thin nanosheets (with low electron contrast) can be detected. High resolution TEM images of a single flake (Figure 2d-f) show highly ordered atomic structures, as also evidenced from the extremely regular fast Fourier transform (FFT) and selected area electron diffraction (SAED) patterns. All spots observed in these patterns could be readily assigned to the [001] zone axis of tetragonal $\mathrm{SnO}$, showing predominant distances between lattice planes of $2.71 \AA$ and $1.91 \AA$, which can be indexed to the (110) and (200) planes respectively. Collectively, these results confirm the highly crystalline nature of the exfoliated $\mathrm{SnO}$, and corroborate the $\mathrm{XRD}$ results showing that the nanosheets, which lay flat on the TEM grid, primarily expose the $(00 z)$ family of lattice planes. 


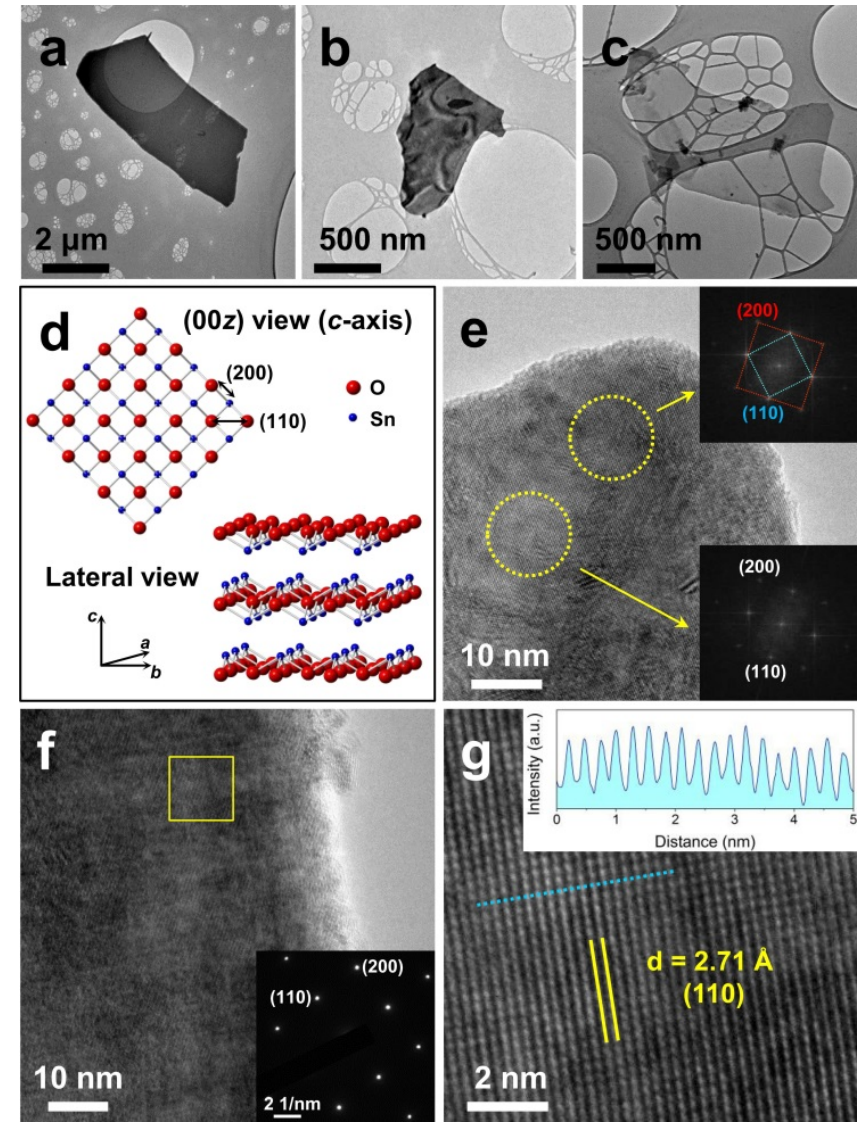

Figure 2. TEM analyses of exfoliated $\mathrm{SnO}$ nanosheets in low resolution (a-c) and high resolution (e-g). Panel (d) shows the "ball and stick" model of the $\mathrm{SnO}$ crystalline structure. The insets in (e) show the fast Fourier transform (FFT) of the highlighted yellow circles. The inset in (f) shows the selected area electron diffraction (SAED) pattern. Panel (g) is the enlarged view of the yellow square in panel (e), and the inset shows the peak profile along the dotted blue line.

To gain further insights into the quality of exfoliated $\mathrm{SnO}$, we used Raman spectroscopy (Figure 3). SnO typically has two main Raman peaks, albeit weak, at 113 and $211 \mathrm{~cm}^{-1}$, corresponding to the $\mathrm{B}_{1 \mathrm{~g}}$ and $\mathrm{A}_{1 \mathrm{~g}}$ vibrational modes, respectively (see also Supporting Information, Figure S6). ${ }^{35}$ These peaks are readily observed in both the as-synthesized SnO (layered microspheres) and in the exfoliated nanosheets. The absence of any additional peaks that may arise from impurities or decomposition products establishes that the soft exfoliation strategy proposed in this study does not influence the intrinsic material properties of SnO. 
However, Raman analysis of a number of individual $\mathrm{SnO}$ microspheres and exfoliated flakes revealed a change in the relative intensity of the $\mathrm{B}_{1 \mathrm{~g}}$ and $\mathrm{A}_{1 \mathrm{~g}}$ peaks between microspheres and nanosheets (Supporting Information, Figure S7). Specifically, the $A_{1 g} / B_{1 g}$ ratio is greater than 1 in thin nanosheets (typically in the range of $1.5-2.4$, even if sometimes the $\mathrm{B}_{1 \mathrm{~g}}$ peak is almost suppressed), while it is smaller than 1 in microspheres (typically between 0.6 and 0.9 ). This distinctive behavior of few-layered $\mathrm{SnO}$ with respect to bulk has also been observed recently for vacuum-deposited $\mathrm{SnO} .{ }^{21}$ This evidence may indicate that the relative intensities of these two Raman modes could be used to distinguish $\mathrm{SnO}$ nanosheets from bulk SnO. However, we note that Raman modes of highly anisotropic structures, and especially 2D materials, have been shown to be sensitive to light polarization and crystal orientation, and therefore the thickness of the nanosheets might not be the only factor affecting the ratio between $\mathrm{A}_{1 \mathrm{~g}}$ and $\mathrm{B}_{1 \mathrm{~g}}$ peaks. ${ }^{36}$ In addition, we observed a progressive decomposition of $\mathrm{SnO}$ (both microspheres and exfoliated nanosheets) when conducting the Raman measurements with laser powers above 5 $\mathrm{mW}$ at $532 \mathrm{~nm}$. This was evidenced by the progressive weakening of the two $\mathrm{SnO}$ modes identified earlier and by the concurrent appearance of additional Raman signatures in the 100$180 \mathrm{~cm}^{-1}$ range (Supporting Information, Figure S8). This finding highlights the importance of tuning the acquisition parameters to ensure that the pristine material properties are preserved, and to avoid misleading results. A two-dimensional Raman map of a $\mathrm{SnO}$ flake shows good match with its optical image (Figure $3 \mathrm{~b}$ and $\mathrm{c}$ ), confirming that the Raman signal is indeed arising from the $\mathrm{SnO}$.

Atomic force microscopy (AFM) was also used to characterize the $\mathrm{SnO}$ nanosheets. Figure $3 \mathrm{~d}$ shows an AFM image of a single flake with a lateral size of approximately $0.5 \mu \mathrm{m}$ and a height of 4.5-5 nm, as confirmed by a line scan (Figure 3e). Considering that the theoretically expected thickness of a $\mathrm{SnO}$ monolayer is $0.484 \mathrm{~nm}$, we can deduce that the flake is composed of $\sim 10$ layers of $\mathrm{SnO} .{ }^{26}$ However, thinner and smaller flakes can also be observed, 
with height below $2 \mathrm{~nm}$, which correspond to $\sim 4$ layers of SnO. These thin flakes could potentially show distinctive properties due to quantum confinement, as already reported for other 2D materials including phosphorene ${ }^{37}$ and $\mathrm{MoS}_{2} \cdot{ }^{38}$ Thicker (30-40 nm) SnO platelets are also observed (Figure 3f,g), possibly resulting from incomplete exfoliation of the layered microspheres. To prove that the AFM images are indicative of $\mathrm{SnO}$, and therefore to exclude the presence of contaminants that could affect the surface morphology of the samples, we performed Raman and AFM analyses on the same spot (Figure S9 in the Supporting Information). We detected $\mathrm{SnO}$ Raman signals in correspondence of the nanosheets observed in the AFM, confirming that the structures observed in the AFM images are indeed $\mathrm{SnO}$ nanosheets.
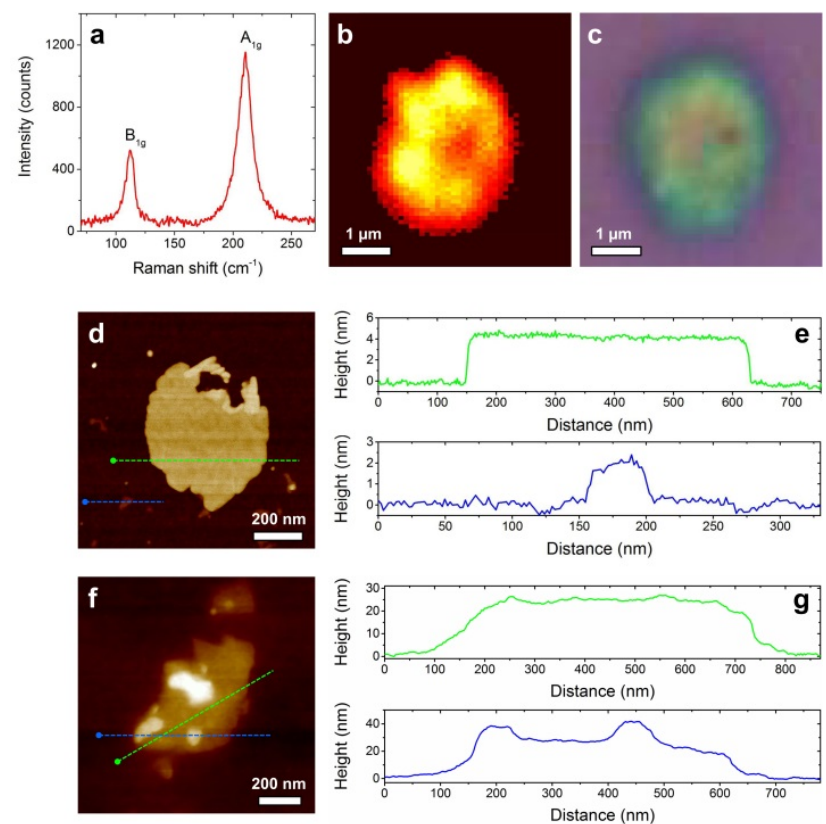

Figure 3. a) Raman spectra of a single SnO flake. b) Raman map and c) corresponding optical microscope image of a typical $\mathrm{SnO}$ flake. d) AFM image of thin $\mathrm{SnO}$ nanosheets. e) Height profile scan along the green and blue dashed lines in (d). f) AFM image of a thick SnO nanosheet. g) Height profile scan along the green and blue dashed lines in (f) 
In order to further investigate the properties of 2D SnO according to its dimensionality, we separated different populations of nanosheets by applying controlled centrifugation conditions (see the Methods section) as reported previously for other 2D materials. ${ }^{39,40}$ While it is typically difficult to precisely separate $2 \mathrm{D}$ materials according to their dimensions (thickness and lateral size) using centrifugation-based methods, these methods do allow a series of samples of progressively reduced dimensions to be isolated when increasing the centrifugation speed. All the samples obtained at different centrifugation speeds were initially analyzed using TEM (Supporting Information, Figure S10). As expected, SnO nanosheets isolated at low centrifugation speeds are typically larger and thicker, as evident from their high electron contrast in the TEM images. However, very thin nanosheets (with low electron contrast) are also observed in these samples. By increasing the centrifugal force, larger and thicker, i.e. heavier flakes are progressively eliminated from the solutions until only very thin and small nanosheets remain. These are sometimes very hard to distinguish because they tend to wrinkle and wrap around each other, and their electron contrast is very similar to the underlying carbon coating of the TEM grid, which is an indication of their near-atomic thicknesses. AFM measurements on SnO flakes revealed that in general, the flakes obtained at low centrifugation speeds (1000-2000 rpm) were highly polydisperse, with thickness up to 40 $\mathrm{nm}$, while those obtained at high speeds $(12000 \mathrm{rpm})$ were predominantly with in the $2-5 \mathrm{~nm}$ range of thicknesses, which corresponds to 4-10 atomic monolayers (additional AFM images in Supporting Information, Figure S11).

After confirming the reduction in size of $\mathrm{SnO}$ with increasing the centrifugal force applied to the sample, we assessed the optical properties of $2 \mathrm{D} \mathrm{SnO}$ as a function of the centrifugation speeds (Figure 4 and Supporting Information, Figure S12). At low speeds, solutions are yellow in color and slightly scattering, corroborating the presence of large and/or thick flakes within the suspension. By increasing the centrifugation speed, the solutions 
become progressively less scattering and less colored. The prominent yellow color of the exfoliated $\mathrm{SnO}$ can be ascribed to its bulk direct band gap with an associated energy of $\sim 2.8$ $\mathrm{eV}$, as confirmed by the Tauc plots presented in the Supporting Information in Figure S13.

The black color of the as-synthesized powders ( $\mathrm{SnO}$ microspheres) is due to the low indirect band gap of $\mathrm{SnO}(\sim 0.7 \mathrm{eV}),{ }^{19,20}$ which is predominant for bulk, micrometer-sized materials, and becomes negligible for dilute nano-sized materials.

By measuring the absorbance of $\mathrm{SnO}$ samples both in standard configuration and within an integrating sphere (see Methods), and by analyzing the tin concentration in each individual solution using atomic emission spectroscopy (Table S2), we obtained absorption, extinction, and scattering plots as a function of wavelength. Briefly, the extinction spectra are obtained from standard transmittance $(T)$ data using the well-known Lambert-beer law in the form of $T=10^{-\varepsilon C l}$, where $\varepsilon$ is the molar extinction coefficient, $C$ is the molar concentration of $\mathrm{SnO}$, and $l$ is the cuvette path length. However, the extinction data encompasses the contribution from both absorption $(\alpha)$ and scattering $(\sigma)$, according to the relationship $\varepsilon(\lambda)=\alpha(\lambda)+\sigma(\lambda)$, where $\lambda$ denotes the wavelength dependence of these functions. ${ }^{40,41}$ By inserting the $\mathrm{SnO}$ samples within an integrating sphere we also measured the pure absorptive component of the samples. The scattering contribution is then evaluated by simply subtracting the absorption from the extinction. As expected, the scattering component decreases with increasing the centrifugation speed, due to the progressive removal of larger sheets from the suspensions. The direct band gap component of $\mathrm{SnO}$ is observed at $\sim 2.8 \mathrm{eV}$ in the samples centrifuged up to $6000 \mathrm{rpm}$, but it cannot be detected when applying higher centrifugation speeds. Interestingly, another sharp absorption edge can be identified at higher energies $(\sim 4.1 \mathrm{eV})$ in all samples, which seems rather independent from centrifugation speed. This distinctive UV absorption, which is 
supported by recent density functional theory (DFT) calculations, is ascribed to band gap widening in quantum confined, atomically thin $\mathrm{SnO}$ sheets. ${ }^{42}$

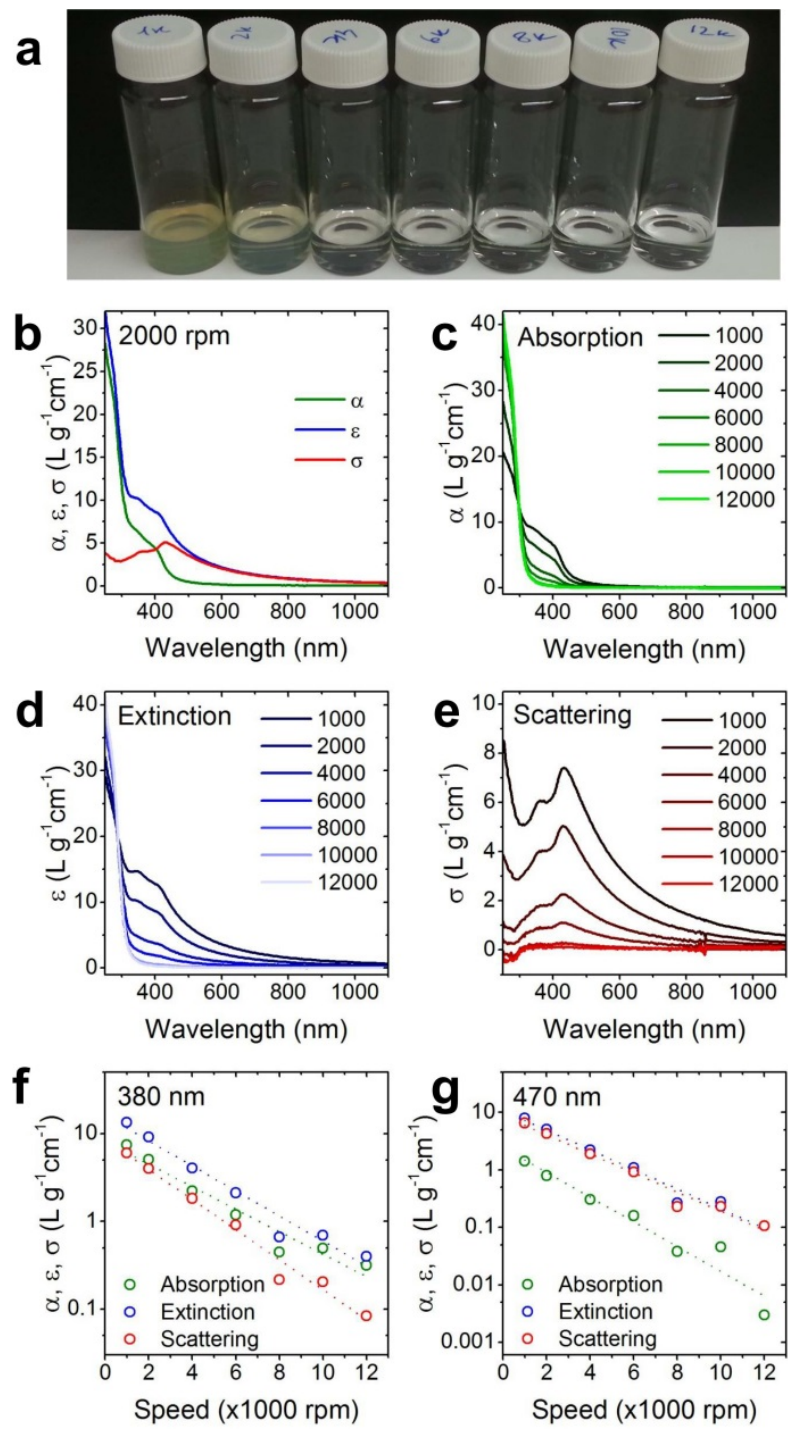

Figure 4. a) Digital photograph of the $\mathrm{SnO}$ suspensions centrifuged at different speeds (1000 rpm to $12000 \mathrm{rpm}$, left to right). b) Absorption, extinction and scattering plots for the sample centrifuged at $2000 \mathrm{rpm}$. c) Absorption, d) extinction and e) scattering plots as a function of centrifugation speed. f,g) Linear-logarithmic plots of absorption, extinction and scattering coefficient at two fixed wavelengths as a function of centrifugation speed.

The optical absorption characteristics as a function of centrifugation conditions are further validated by photoluminescence (PL) measurements (Figure 5 and Figure S14). All 
samples show two main emission peaks, one centered at $313 \mathrm{~nm}(3.97 \mathrm{eV}$, peak 1) and another one centered at $407 \mathrm{~nm}(3.05 \mathrm{eV}$, peak 2). The first peak can be ascribed to the band edge emission of quantum confined sheets, corresponding to the sharp absorption observed at 4.1 $\mathrm{eV}$, while the second peak, which is much broader, is possibly due to surface defects and trap state emissions that are commonly observed in many semiconducting nanomaterials. ${ }^{43,44,45}$ Similar PL spectra of nanostructured SnO showing two separate contributions - a sharp UV peak and a broad peak in the blue range - have also been observed recently. ${ }^{46}$ Interestingly, at low centrifugation speeds the broad emission peak in the visible region shows an additional low energy contribution centered in the $425-450 \mathrm{~nm}$ range $(2.75-2.9 \mathrm{eV}$, peak 3$)$, which is ascribed to the bulk direct band gap discussed earlier. We note that the evaluation of the band gap using both the absorption edge and the PL peak is an approximation of the actual band gap value. Specifically, while the Tauc analysis is fairly accurate for band-to-band transitions in bulk semiconductors, it fails to precisely identify excitonic transitions in highly quantum confined materials, due to size-dependent variation in exciton binding energies and dielectric screening. Conversely, PL spectra are representative of the recombination of the excitons, but they are also affected by other parameters such as non radiative relaxations and trap states. However, when applied to our $\mathrm{SnO}$ samples both techniques identify a high energy component (4-4.1 eV), ascribed to highly quantum confined $\mathrm{SnO}$, and a lower energy component (2.75$2.9 \mathrm{eV}$ ), which is consistent with literature values for the direct band gap transition in bulk $\mathrm{SnO}$.

Overall, these optical measurements support (i) the presence of highly quantum confined $\mathrm{SnO}$ thin sheets in all samples, and (ii) the additional presence of thicker nanosheets and platelets in samples separated at low centrifugation speeds, which are responsible for the lower energy absorption/emission components, and for the scattering contribution. 

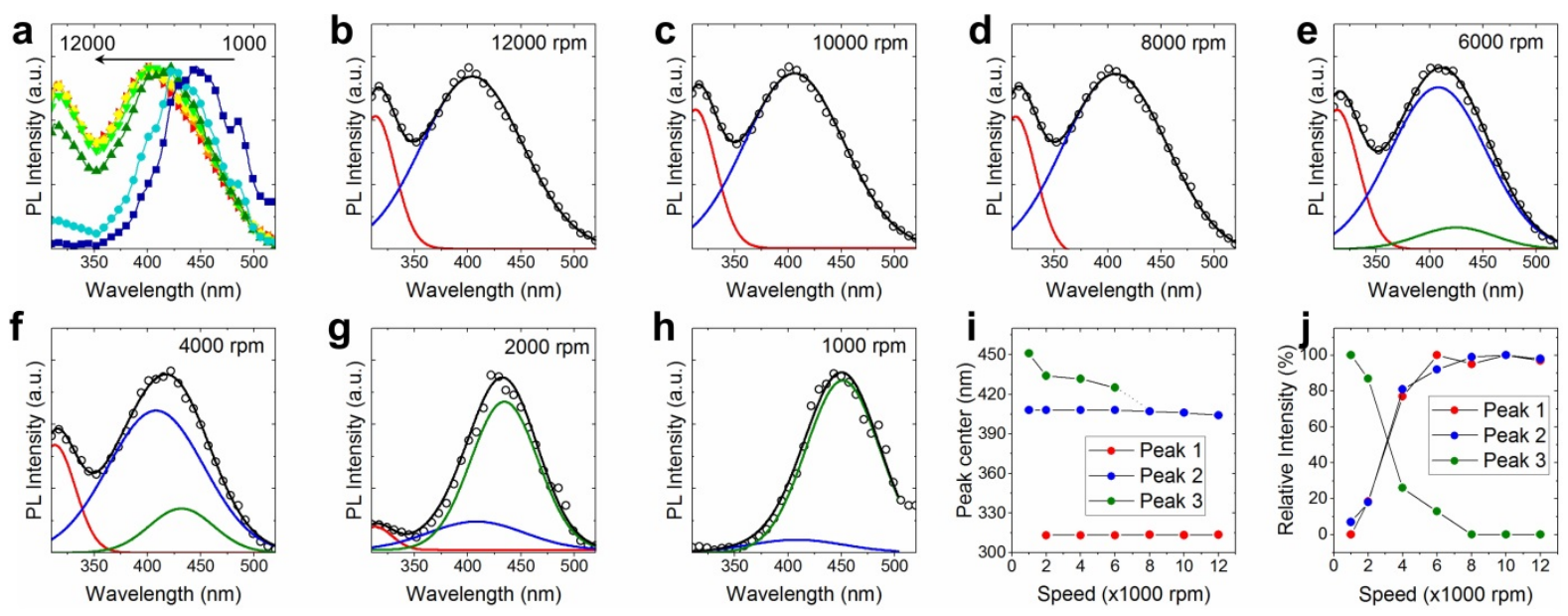

Figure 5. a) Photoluminescence (PL) spectra of $\mathrm{SnO}$ suspensions at different centrifugation speeds. b-h) Deconvolution of PL spectra into three separate contributions fitted with Gaussian functions. Plot of the peak maxima (i) and the relative peak intensities (j) of these three contributions as a function of the centrifugation speed.

Given the observed variations in optical properties according to centrifugation speed, we further explored the energy levels of $\mathrm{SnO}$ through the use of photoelectron spectroscopy in air (PESA, Supporting Information, Figure S15). This technique enables to measure the ionization potential of materials, and to evaluate the work function and the energy level associated with the valence band maximum (for metallic and semiconducting samples, respectively). ${ }^{47}$ The layered $\mathrm{SnO}$ microspheres have a valence band maximum of $4.4 \mathrm{eV}$, in line with both theoretical ${ }^{42}$ and experimental ${ }^{20}$ reported values for $\mathrm{SnO}$. The exfoliated $\mathrm{SnO}$ samples show a very similar valence band maximum at 4.4-4.5 eV. Notably, at higher centrifugation speeds the top of the valence band is seen to shift towards deeper values, especially in the samples centrifuged at $8000 \mathrm{rpm}$ or above, although the signal-to-noise ratio in these samples is rather low due to the small amounts of material probed. Optical absorption measurements show that these samples do not contain thick flakes that would have otherwise been responsible for optical absorptions in the visible range, but they all possess a wide band gap associated with quantum confined sheets. In light of this, we can conclude that thick 
exfoliated flakes show bulk-like properties (direct band gap energy and valence band maximum similar to micron-sized $\mathrm{SnO}$ ), while highly quantum confined sheets show a wider band gap and a deeper valence band maximum, which is consistent with recent theoretical predictions for atomically thin $\mathrm{SnO}$ nanosheets. ${ }^{42}$ These thin $\mathrm{SnO}$ nanosheets are responsible for the distinctive optical and electronic properties observed in the samples separated at high centrifugation speeds.

To assess the potential applicability of $2 \mathrm{D} \mathrm{SnO}$ as a semiconducting material for optoelectronics, and to further demonstrate the different properties of bulk-like and quantum confined nanosheets, we evaluated the electrical response of the SnO flakes under photoexcitation. Two sets of SnO nanosheets were separated at either $1000 \mathrm{rpm}$ or $12000 \mathrm{rpm}$ and were deposited between two pre-patterned Au electrodes as described in the Methods section. As presented earlier, samples separated at high centrifugation speeds are composed only of quantum confined nanosheets which can absorb only UV light with wavelengths shorter than $\sim 300 \mathrm{~nm}$ (estimated band gap $>4 \mathrm{eV}$ ). Conversely, exfoliated $\mathrm{SnO}$ nanosheets separated at low centrifugation speeds also show a prominent absorption at $\sim 2.8 \mathrm{eV}$, corresponding to the bulk direct band gap. These nanosheets can absorb light in the blue region, with wavelengths up to $\sim 450 \mathrm{~nm}$. Therefore, the photoresponse of the "thick" flakes is expected to be radically different compared to that of the thin, quantum confined nanosheets. Figure 6 shows the absorption spectra of the two families of $\mathrm{SnO}$ nanosheets, together with the emission spectra of the UV (peak at $280 \mathrm{~nm}$ ) and blue (peak at $455 \mathrm{~nm}$ ) LEDs that we used to probe the optoelectronic responses of $\mathrm{SnO}$ devices. Thicker nanosheets separated at $1000 \mathrm{rpm}$ can absorb light emitted by both LEDs, while quantum confined $\mathrm{SnO}$ obtained at high centrifugation speeds can only absorb the high energy UV radiation. It was observed that the current through the device comprising the thick flakes increased during exposure to both light sources (Figure 6b), while in the case of the quantum confined nanosheets, the current increased only on exposure to UV 
light and no discernible response was seen under blue light excitation (Figure 6d). Moreover, the photocurrent measured when a thick flake is exposed to UV light is higher compared to that measured when the same flake is exposed to blue light ( $\sim 60 \%$ increase compared to the baseline value under $\mathrm{UV}$, with respect to $\sim 30 \%$ increase under blue light). This is in agreement with the lower energy associated with visible light compared to UV light, and the lower absorption cross section of $\mathrm{SnO}$ at visible wavelengths (see Figure 6a). These results correlate well with the optical properties presented earlier and highlight the distinctive change in the intrinsic characteristics of $2 \mathrm{D} \mathrm{SnO}$ when it reaches the quantum confinement regime.
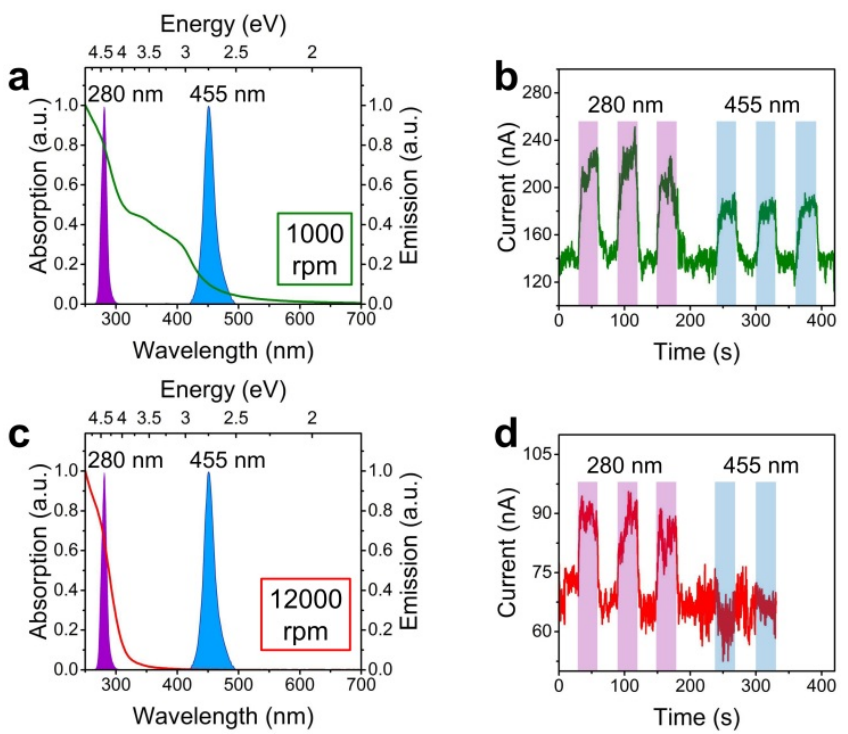

Figure 6. a,c) Absorption spectra for $\mathrm{SnO}$ nanosheets obtained after centrifugation at $1000 \mathrm{rpm}$ (a) and $12000 \mathrm{rpm}$ (c). The emission spectra for the UV (280 nm) and blue (455 nm) LEDs used for the photoconductivity measurements are shown as well. b,d) Current output at an applied voltage of $2 \mathrm{~V}$ for $\mathrm{SnO}$ nanosheets obtained after centrifugation at $1000 \mathrm{rpm}$ (b) and $12000 \mathrm{rpm}$ (d) during exposure to UV and blue LEDs. Three on/off cycles are presented except for the $12000 \mathrm{rpm}$ (thin) nanosheets exposed to blue light, because no photoresponse was detected.

\section{Conclusion}


In conclusion, we have presented a new "soft" exfoliation strategy to successfully exfoliate $\mathrm{SnO}$ nanosheets composed of $<10$ atomic monolayers. This strategy relies on producing highly layered $\mathrm{SnO}$ microspheres using tin (II) 2-ethyl hexanoate and triethylamine. This morphology enables the fast and easy exfoliation of $\mathrm{SnO}$ nanosheets through simple sonication at low powers and in common solvents such as ethanol. Highly crystalline nanosheets with lateral size ranging between hundreds of nanometers and few micrometers, and thickness as low as 2 nanometers are readily achieved. Controlled centrifugation is then applied to separate different populations of nanosheets, which possess size-tunable optical and electronic properties, including widening of the band gap, indicating a strong quantum confinement effect in $2 \mathrm{D}$ SnO. Photoconductivity measurements demonstrate a strong correlation between the quantumconfined properties of thin $\mathrm{SnO}$ nanosheets and their selective photoelectrical response in the high energy UV light, therefore indicating the potential of few monolayer thick SnO for optoelectronic applications under specific UV light conditions. These results highlight the importance of carefully designing the starting materials that can then be easily exfoliated to produce their $2 \mathrm{D}$ counterparts. The strategy proposed here constitutes a step forward to developing reliable and scalable methods for the production of 2D materials, which can then be used for the fabrication of next-generation (opto)electronic devices.

\section{Methods}

\section{Materials}

Tin (II) 2-ethyl hexanoate (SnHex, 92.5-100\%), triethylamine (TEA, >99\%) and N,Ndimehtylformamide (DMF, anhydrous 99.8\%) were supplied by Sigma Aldrich. Ethanol $(>99.5 \%)$, acetone $(>99 \%)$, and diethyl ether ( $>99.5 \%)$ were purchased from Chem-Supply. 
In a standard reaction, SnHex $(5 \mathrm{~mL})$ and TEA $(2 \mathrm{~mL})$ are mixed at room temperature and vigorously stirred for 2 minutes triggering a mild exothermic reaction. Separately, a vial containing $9 \mathrm{~mL}$ of deionized (Milli-Q) water is preheated in a water bath at $40{ }^{\circ} \mathrm{C}$ and the SnHex-TEA mixture is rapidly injected into the water under stirring causing the instantaneous precipitation of tin oxide hydroxide $\left(\mathrm{Sn}_{6} \mathrm{O}_{4}(\mathrm{OH})_{4}\right)$ in the form of a white powder. After 15 minutes the flask is removed from the water bath, letting the solid phase sediment at the bottom of the vial. The liquid supernatant is then removed, and the flask is placed in a preheated oven at $180{ }^{\circ} \mathrm{C}$ for 60 minutes causing the conversion of $\mathrm{Sn}_{6} \mathrm{O}_{4}(\mathrm{OH})_{4}$ to $\mathrm{SnO}$ microspheres in the form of a black powder. The resulting powder is then suspended in a mixture of acetone and ethanol and centrifuged at $4000 \mathrm{rpm}$ for 5 minutes to remove the unreacted precursors and excess organics. This procedure is repeated 3 times, and then an additional wash cycle is done with diethyl ether. The obtained $\mathrm{SnO}$ powders are dried overnight in vacuum and stored in the dark.

Exfoliation of $\mathrm{SnO}$ powders is conducted by suspending $160 \mathrm{mg}$ of the synthesized powders in $40 \mathrm{~mL}$ ethanol and by sonicating the suspension in a benchtop ultrasonic bath (Unisonics FXP4M with operating power of $40 \mathrm{~W}$ ) for 5 minutes. Within a few seconds, the suspension becomes yellow and slightly scattering, confirming the successful exfoliation of SnO. The suspension is then centrifuged at $500 \mathrm{rpm}$ for 5 minutes to remove any unexfoliated powder, and the supernatant is split into seven tubes and centrifuged at different speeds $(1000,2000$, $4000,6000,8000,10000$ or $12000 \mathrm{rpm}$ for 5 minutes). Of each tube, only the supernatant is kept and the pellet is discarded. The obtained samples are then stored in the dark for future use.

\section{Characterization techniques}

Unless specified differently, all characterizations were performed on samples deposited on silicon substrates by simply drop-casting the $\mathrm{SnO}$ nanosheet solutions and letting the solvent evaporate naturally. X-ray diffraction (XRD) patterns were collected on samples deposited on 
glass substrates using a Bruker D4 Endeavor diffractometer equipped with a $\mathrm{Cu}-\mathrm{K} \alpha$ radiation source and operated at $40 \mathrm{kV}$ and $35 \mathrm{~mA}$. Thermogravimetric analysis (TGA) of powder samples was conducted on a Perkin Elmer Pyris 1 instrument at a heating rate of $5{ }^{\circ} \mathrm{C} / \mathrm{min}$. Scanning electron microscopy (SEM) images were acquired on a FEI Nova 200 NanoSEM operated at $10 \mathrm{kV}$ and $30 \mathrm{pA}$. Transmission electron microscopy (TEM) images were acquired on a Jeol 1010 microscope operated at $100 \mathrm{kV}$. High resolution (HR) TEM images were acquired on a Jeol $2100 \mathrm{~F}$ microscope operated at $80 \mathrm{kV}$. For TEM analyses, samples were prepared by depositing a drop of the nanosheet solutions on a holey carbon-coated copper grid. X-ray photoelectron spectroscopy (XPS) was conducted on a Thermo Scientific K-Alpha instrument using an Al Ka x-ray source (1486.7 eV). Samples were deposited on Au-coated Si substrates and the core level XPS spectra of the target elements ( $\mathrm{Sn} 3 \mathrm{~d}$ and O 1s) were obtained and corrected for charging effect. All spectra were corrected using Shirley background correction and resolved using standard Gaussian-Lorentzian functions. Raman spectra were acquired on a Horiba LabRAM HR Evolution Raman spectrometer using a $532 \mathrm{~nm}$ laser and power ranging between 0.5 and $50 \mathrm{~mW}$ (1-100\% of the total laser power) using either pure $\mathrm{Si}$ or $\mathrm{Si} / \mathrm{SiO}_{2}$ (300 nm of thermally grown oxide) as substrates. Atomic Force Microscopy (AFM) images were collected using a Bruker Dimension Icon instrument in tapping mode. The chemical composition of the samples centrifuged at different speeds was analyzed with atomic emission spectroscopy (AES) using an Agilent 4200 MP-AES instrument after digesting the solution in concentrated hydrochloric acid. Optical absorption spectra of nanosheet suspensions in the UV-Vis-NIR range were conducted on a Perkin Elmer Lambda 1050 spectrophotometer equipped with an integrating sphere. The extinction spectra of $\mathrm{SnO}$ suspensions were acquired in standard configuration, while the absorption component of the extinction was obtained by inserting the cuvettes with the $\mathrm{SnO}$ suspensions within the integrating sphere, allowing for the simultaneous collection of both transmitted and scattered 
radiation. The scattering component was then obtained by subtracting the absorption from the extinction. Photoluminescence (PL) spectra were obtained on a Perkin Elmer LS50 fluorimeter using an excitation wavelength of $280 \mathrm{~nm}$. Photoelectron spectroscopy in air (PESA) was conducted on a Riken Keiki AC-2 spectrometer using $50 \mathrm{nW}$ radiation on samples deposited on glass substrates. The electrical measurements were conducted on $\mathrm{SnO}$ nanosheets dropcasted onto pre-patterned electrodes. The patterns were created on Si substrates covered with $100 \mathrm{~nm} \mathrm{SiO}$ : a photoresist layer was deposited by spin coating and dried at $100{ }^{\circ} \mathrm{C}$, exposed to UV light through a mask using a mask aligner system (MA6, SUSS MicroTech) which defined the patterns, and subsequently developed. The Cr/Au metal electrodes (10/100 nm) were then deposited on the developed patterns using electron beam evaporation. Finally, the lift-off in acetone was carried out to reveal the required metallic contact pads for microprobes and electrical measurement. The electrical measurements were conducted under ambient air and at room temperature using a Keithley 4200SCS semiconductor parameter analyser. The optical stimulation is provided by using commercial high power light-emitting diodes (LED, Thorlabs, Inc.). The illumination power was calibrated at $2.5 \mathrm{~mW} / \mathrm{cm}^{2}$ using a commercial UV-enhanced silicon photodetector (Newport Corporation).

\section{Author Contributions}

MS and EDG contributed equally to this work. The manuscript was written through contributions of all authors. All authors have given approval to the final version of the manuscript.

\section{Acknowledgement}

MS thanks the Commonwealth of Australia for an Australian Postgraduate Award. EDG, RR and SW thank RMIT University for the Vice Chancellor Research Fellowships scheme. The 
Australian Research Council is acknowledged for supporting this work through the following grants and fellowships: Future Fellowship (FT140101285, VB), Discovery Project (DP170103477, VB and RR), Discovery Early Career Researcher Award (DE170100164, EDG), Linkage Infrastructure, Equipment and Facilities grant (LE150100001). The authors acknowledge the facilities and the technical assistance of the RMIT University's Microscopy and Microanalysis Facility (RMMF). The authors would also like to thank the CSIRO for providing access to absorption spectrophotometer and PESA.

\section{References}

1. Novoselov, K. S.; Mishchenko, A.; Carvalho, A.; Castro Neto, A. H., 2D materials and van der Waals heterostructures. Science 2016, 353 (6298).

2. Liu, Y.; Weiss, N. O.; Duan, X.; Cheng, H.-C.; Huang, Y.; Duan, X., Van der Waals heterostructures and devices. Nature Reviews Materials 2016, 1, 16042.

3. Novoselov, K. S.; Geim, A. K.; Morozov, S. V.; Jiang, D.; Zhang, Y.; Dubonos, S. V.; Grigorieva, I. V.; Firsov, A. A., Electric Field Effect in Atomically Thin Carbon Films. Science 2004, 306 (5696), 666-669.

4. Hernandez, Y.; Nicolosi, V.; Lotya, M.; Blighe, F. M.; Sun, Z.; De, S.; McGovern, I. T.; Holland, B.; Byrne, M.; Gun'Ko, Y. K.; Boland, J. J.; Niraj, P.; Duesberg, G.; Krishnamurthy, S.; Goodhue, R.; Hutchison, J.; Scardaci, V.; Ferrari, A. C.; Coleman, J. N., High-yield production of graphene by liquid-phase exfoliation of graphite. Nat Nano 2008, 3 (9), 563-568.

5. Wang, Q. H.; Kalantar-Zadeh, K.; Kis, A.; Coleman, J. N.; Strano, M. S., Electronics and optoelectronics of two-dimensional transition metal dichalcogenides. Nat Nano 2012, 7 (11), 699-712.

6. $\quad$ Gong, Y.; Lin, J.; Wang, X.; Shi, G.; Lei, S.; Lin, Z.; Zou, X.; Ye, G.; Vajtai, R.; Yakobson, B. I.; Terrones, H.; Terrones, M.; Tay, Beng K.; Lou, J.; Pantelides, S. T.; Liu, Z.; Zhou, W.; Ajayan, P. M., Vertical and in-plane heterostructures from WS2/MoS2 monolayers. Nat Mater 2014, 13 (12), 1135-1142.

7. Xiao, X.; Song, H.; Lin, S.; Zhou, Y.; Zhan, X.; Hu, Z.; Zhang, Q.; Sun, J.; Yang, B.; Li, T.; Jiao, L.; Zhou, J.; Tang, J.; Gogotsi, Y., Scalable salt-templated synthesis of twodimensional transition metal oxides. Nature Communications 2016, 7, 11296.

8. ten Elshof, J. E.; Yuan, H.; Gonzalez Rodriguez, P., Two-Dimensional Metal Oxide and Metal Hydroxide Nanosheets: Synthesis, Controlled Assembly and Applications in Energy Conversion and Storage. Advanced Energy Materials 2016, 6 (23), 1600355-n/a.

9. Anasori, B.; Lukatskaya, M. R.; Gogotsi, Y., 2D metal carbides and nitrides (MXenes) for energy storage. Nature Reviews Materials 2017, 2, 16098.

10. Balendhran, S.; Walia, S.; Nili, H.; Sriram, S.; Bhaskaran, M., Elemental Analogues of Graphene: Silicene, Germanene, Stanene, and Phosphorene. Small 2015, 11 (6), 640-652. 
11. Sumeet, W.; Ylias, S.; Taimur, A.; Matthew, R. F.; Rajesh, R.; Aram, A.; Suresh, K. B.; Sharath, S.; Madhu, B.; Vipul, B.; Sivacarendran, B., Defining the role of humidity in the ambient degradation of few-layer black phosphorus. 2D Materials 2017, 4 (1), 015025.

12. Akkerman, Q. A.; Motti, S. G.; Srimath Kandada, A. R.; Mosconi, E.; D'Innocenzo, V.; Bertoni, G.; Marras, S.; Kamino, B. A.; Miranda, L.; De Angelis, F.; Petrozza, A.; Prato, M.; Manna, L., Solution Synthesis Approach to Colloidal Cesium Lead Halide Perovskite Nanoplatelets with Monolayer-Level Thickness Control. Journal of the American Chemical Society 2016, 138 (3), 1010-1016.

13. Balendhran, S.; Deng, J.; Ou, J. Z.; Walia, S.; Scott, J.; Tang, J.; Wang, K. L.; Field, M. R.; Russo, S.; Zhuiykov, S.; Strano, M. S.; Medhekar, N.; Sriram, S.; Bhaskaran, M.; Kalantar-zadeh, K., Enhanced Charge Carrier Mobility in Two-Dimensional High Dielectric Molybdenum Oxide. Advanced Materials 2013, 25 (1), 109-114.

14. Sun, Z.; Liao, T.; Dou, Y.; Hwang, S. M.; Park, M.-S.; Jiang, L.; Kim, J. H.; Dou, S. $\mathrm{X}$., Generalized self-assembly of scalable two-dimensional transition metal oxide nanosheets. Nature Communications 2014, 5, 3813.

15. Bai, Y.; Xing, Z.; Yu, H.; Li, Z.; Amal, R.; Wang, L., Porous Titania Nanosheet/Nanoparticle Hybrids as Photoanodes for Dye-Sensitized Solar Cells. ACS Applied Materials \& Interfaces 2013, 5 (22), 12058-12065.

16. Okamoto, Y.; Ida, S.; Hyodo, J.; Hagiwara, H.; Ishihara, T., Synthesis and Photocatalytic Activity of Rhodium-Doped Calcium Niobate Nanosheets for Hydrogen Production from a Water/Methanol System without Cocatalyst Loading. Journal of the American Chemical Society 2011, 133 (45), 18034-18037.

17. Alsaif, M. M. Y. A.; Latham, K.; Field, M. R.; Yao, D. D.; Medehkar, N. V.; Beane, G. A.; Kaner, R. B.; Russo, S. P.; Ou, J. Z.; Kalantar-zadeh, K., Tunable Plasmon Resonances in Two-Dimensional Molybdenum Oxide Nanoflakes. Advanced Materials 2014, 26 (23), 39313937.

18. Deliang, C.; Xianxiang, H.; Hejing, W.; Yu, W.; Hailong, W.; Xinjian, L.; Rui, Z.; Hongxia, L.; Hongliang, X.; Shaokang, G.; Jing, S.; Lian, G., The enhanced alcohol-sensing response of ultrathin WO 3 nanoplates. Nanotechnology 2010, 21 (3), 035501.

19. Ogo, Y.; Hiramatsu, H.; Nomura, K.; Yanagi, H.; Kamiya, T.; Hirano, M.; Hosono, H., p-channel thin-film transistor using p-type oxide semiconductor, SnO. Applied Physics Letters 2008, 93 (3), 032113.

20. Quackenbush, N. F.; Allen, J. P.; Scanlon, D. O.; Sallis, S.; Hewlett, J. A.; Nandur, A. S.; Chen, B.; Smith, K. E.; Weiland, C.; Fischer, D. A.; Woicik, J. C.; White, B. E.; Watson, G. W.; Piper, L. F. J., Origin of the Bipolar Doping Behavior of SnO from X-ray Spectroscopy and Density Functional Theory. Chemistry of Materials 2013, 25 (15), 3114-3123.

21. Saji, K. J.; Tian, K.; Snure, M.; Tiwari, A., 2D Tin Monoxide-An Unexplored p-Type van der Waals Semiconductor: Material Characteristics and Field Effect Transistors. Advanced Electronic Materials 2016, 2 (4), 1500453-n/a.

22. Nomura, K.; Kamiya, T.; Hosono, H., Ambipolar Oxide Thin-Film Transistor. Advanced Materials 2011, 23 (30), 3431-3434.

23. Liang, L.; Sun, Y.; Lei, F.; Gao, S.; Xie, Y., Free-floating ultrathin tin monoxide sheets for solar-driven photoelectrochemical water splitting. Journal of Materials Chemistry A 2014, 2 (27), 10647-10653.

24. Sakaushi, K.; Oaki, Y.; Uchiyama, H.; Hosono, E.; Zhou, H.; Imai, H., Synthesis and Applications of SnO Nanosheets: Parallel Control of Oxidation State and Nanostructure Through an Aqueous Solution Route. Small 2010, 6 (6), 776-781.

25. Zhang, F.; Zhu, J.; Zhang, D.; Schwingenschlögl, U.; Alshareef, H. N., TwoDimensional SnO Anodes with a Tunable Number of Atomic Layers for Sodium Ion Batteries. Nano Letters 2017, 17 (2), 1302-1311. 
26. Lefebvre, I.; Szymanski, M. A.; Olivier-Fourcade, J.; Jumas, J. C., Electronic structure of tin monochalcogenides from $\mathrm{SnO}$ to SnTe. Physical Review B 1998, 58 (4), 1896-1906.

27. Nicolosi, V.; Chhowalla, M.; Kanatzidis, M. G.; Strano, M. S.; Coleman, J. N., Liquid Exfoliation of Layered Materials. Science 2013, 340 (6139).

28. Backes, C.; Higgins, T. M.; Kelly, A.; Boland, C.; Harvey, A.; Hanlon, D.; Coleman, J. N., Guidelines for Exfoliation, Characterization and Processing of Layered Materials Produced by Liquid Exfoliation. Chemistry of Materials 2016.

29. Uchiyama, H.; Nakanishi, S.; Kozuka, H., Biomimetic synthesis of nanostructured SnO particles from Sn6O4(OH)4 in aqueous solution of gelatin. CrystEngComm 2015, 17 (3), 628632.

30. Della Gaspera, E.; van Embden, J.; Chesman, A. S. R.; Duffy, N. W.; Jasieniak, J. J., Mimicry of Sputtered i-ZnO Thin Films Using Chemical Bath Deposition for SolutionProcessed Solar Cells. ACS Applied Materials \& Interfaces 2014, 6 (24), 22519-22526.

31. Stranick, M. A.; Moskwa, A., SnO by XPS. Surface Science Spectra 1993, 2 (1), 4549.

32. Marrani, A. G.; Caprioli, F.; Boccia, A.; Zanoni, R.; Decker, F., Electrochemically deposited ZnO films: an XPS study on the evolution of their surface hydroxide and defect composition upon thermal annealing. Journal of Solid State Electrochemistry 2014, 18 (2), 505-513.

33. Della Gaspera, E.; Kennedy, D. F.; van Embden, J.; Chesman, A. S. R.; Gengenbach, T. R.; Weber, K.; Jasieniak, J. J., Flash-Assisted Processing of Highly Conductive Zinc Oxide Electrodes from Water. Advanced Functional Materials 2015, 25 (47), 7263-7271.

34. Sinha, A. K.; Manna, P. K.; Pradhan, M.; Mondal, C.; Yusuf, S. M.; Pal, T., Tin oxide with a p-n heterojunction ensures both UV and visible light photocatalytic activity. $R S C$ Advances 2014, 4 (1), 208-211.

35. Geurts, J.; Rau, S.; Richter, W.; Schmitte, F. J., SnO films and their oxidation to SnO2: Raman scattering, IR reflectivity and X-ray diffraction studies. Thin Solid Films 1984, 121 (3), 217-225.

36. Liu, S.; Huo, N.; Gan, S.; Li, Y.; Wei, Z.; Huang, B.; Liu, J.; Li, J.; Chen, H., Thicknessdependent Raman spectra, transport properties and infrared photoresponse of few-layer black phosphorus. Journal of Materials Chemistry C 2015, 3 (42), 10974-10980.

37. Woomer, A. H.; Farnsworth, T. W.; Hu, J.; Wells, R. A.; Donley, C. L.; Warren, S. C., Phosphorene: Synthesis, Scale-Up, and Quantitative Optical Spectroscopy. ACS Nano 2015, 9 (9), 8869-8884.

38. Gan, Z. X.; Liu, L. Z.; Wu, H. Y.; Hao, Y. L.; Shan, Y.; Wu, X. L.; Chu, P. K., Quantum confinement effects across two-dimensional planes in MoS2 quantum dots. Applied Physics Letters 2015, 106 (23), 233113.

39. Khan, U.; O’Neill, A.; Porwal, H.; May, P.; Nawaz, K.; Coleman, J. N., Size selection of dispersed, exfoliated graphene flakes by controlled centrifugation. Carbon 2012, 50 (2), 470-475.

40. $\quad$ Backes, C.; Smith, R. J.; McEvoy, N.; Berner, N. C.; McCloskey, D.; Nerl, H. C.; O’Neill, A.; King, P. J.; Higgins, T.; Hanlon, D.; Scheuschner, N.; Maultzsch, J.; Houben, L.; Duesberg, G. S.; Donegan, J. F.; Nicolosi, V.; Coleman, J. N., Edge and confinement effects allow in situ measurement of size and thickness of liquid-exfoliated nanosheets. Nature Communications 2014, 5, 4576.

41. Hanlon, D.; Backes, C.; Doherty, E.; Cucinotta, C. S.; Berner, N. C.; Boland, C.; Lee, K.; Harvey, A.; Lynch, P.; Gholamvand, Z.; Zhang, S.; Wang, K.; Moynihan, G.; Pokle, A.; Ramasse, Q. M.; McEvoy, N.; Blau, W. J.; Wang, J.; Abellan, G.; Hauke, F.; Hirsch, A.; Sanvito, S.; O'Regan, D. D.; Duesberg, G. S.; Nicolosi, V.; Coleman, J. N., Liquid exfoliation 
of solvent-stabilized few-layer black phosphorus for applications beyond electronics. Nature Communications 2015, 6, 8563.

42. Zhou, W.; Umezawa, N., Band gap engineering of bulk and nanosheet SnO: an insight into the interlayer Sn-Sn lone pair interactions. Physical Chemistry Chemical Physics 2015, 17 (27), 17816-17820.

43. Resch, U.; Eychmueller, A.; Haase, M.; Weller, H., Absorption and fluorescence behavior of redispersible cadmium sulfide colloids in various organic solvents. Langmuir 1992, 8 (9), 2215-2218.

44. Joo, J.; Na, H. B.; Yu, T.; Yu, J. H.; Kim, Y. W.; Wu, F.; Zhang, J. Z.; Hyeon, T., Generalized and Facile Synthesis of Semiconducting Metal Sulfide Nanocrystals. Journal of the American Chemical Society 2003, 125 (36), 11100-11105.

45. Djurišić, A. B.; Choy, W. C. H.; Roy, V. A. L.; Leung, Y. H.; Kwong, C. Y.; Cheah, K. W.; Gundu Rao, T. K.; Chan, W. K.; Fei Lui, H.; Surya, C., Photoluminescence and Electron Paramagnetic Resonance of ZnO Tetrapod Structures. Advanced Functional Materials 2004, 14 (9), 856-864.

46. Sakaushi, K.; Oaki, Y.; Uchiyama, H.; Hosono, E.; Zhou, H.; Imai, H., Aqueous solution synthesis of $\mathrm{SnO}$ nanostructures with tuned optical absorption behavior and photoelectrochemical properties through morphological evolution. Nanoscale 2010, 2 (11), 2424-2430.

47. Jasieniak, J.; Califano, M.; Watkins, S. E., Size-Dependent Valence and Conduction Band-Edge Energies of Semiconductor Nanocrystals. ACS Nano 2011, 5 (7), 5888-5902. 